\title{
Dynamical Properties of Sea Surface Microwave Backscatter at Low-Incidence: Correlation Time and Doppler Shift
}

\author{
Olivier Boisot, Laïba Amarouche, Jean-Claude Lalaurie, and Charles-Antoine Guérin
}

5 Abstract-We investigate some temporal properties of the mi6 crowave backscattered field from the sea surface at low incidence, 7 namely, the decorrelation time and the Doppler shift distribution. 8 These quantities may have an important impact on the perfor9 mances of the altimeter and synthetic aperture radar systems in $10 \mathrm{Ku}$ - and Ka-bands and must be accurately evaluated. In the frame11 work of classical analytical scattering models and for realistic sea 12 spectra, we obtain a simple expression for the decorrelation time 13 with respect to the main sea state parameters and the scattering 14 geometry. We further propose an original approach based on a 15 time-domain estimator to evaluate the distribution of instanta16 neous Doppler shifts and the Doppler centroid. The evolution of 17 the latter with the sea state and scattering angles is calculated and 18 discussed. A procedure is proposed to recover the full two-sided 19 Doppler spectrum. We discuss the use of the Doppler shift in view 20 of the geophysical parameter retrieval at low incidence. We find 21 that the surface wind vector can, in principle, be well estimated 22 from the azimuthal variation of the Doppler shift, whereas the 23 signature of the surface current is not sufficient to allow for its 24 estimation.

25 Index Terms-Correlation time, Doppler shift, low-incidence, 26 microwave ocean remote sensing.

\section{INTRODUCTION}

$28 \mathrm{~N}$ THE last years, there has been a significant improve30 ment of the capabilities of current or forthcoming altimeter 31 missions in terms of resolution and accuracy. Some of these 32 improvements consist in the replacement of the usual cen33 timeter radar wavelength (C- and Ku-bands) with millimeter 34 wavelength (Ka-band). In a conventional altimeter, such as 35 the AltiKa mission, the use of the Ka-band allows to work 36 at higher pulse repetition frequencies (PRFs) due to the faster 37 decorrelation of the backscattered signal and therefore to reach 38 an average waveform within a smaller amount of time with a 39 correct speckle noise amplitude. In the wide-swath altimetry

Manuscript received May 25, 2016; revised July 22, 2016; accepted July 22, 2016. This work was supported in part by the Centre National d'Études Spatiales and in part by the Collecte Localisation Satellite.

$\mathrm{O}$. Boisot is with the Mediterranean Institute of Oceanography, University Toulon, Aix Marseille University, CNRS, IRD, MIO UM 110 La Garde, France, and also with the Office National d'Etudes et de Recherches Aérospatiales (ONERA), Salon-de-Provence, France (e-mail: olivier.boisot@univ-tln.fr).

L. Amarouche and J.-C. Lalaurie are with Collecte Localisation Satellite and Centre National d'Études Spatiales, 31400 Toulouse, France (e-mail: lamarouche@cls.fr; Jean-Claude.Lalaurie@cnes.fr).

C.-A. Guérin is with the Mediterranean Institute of Oceanography, University Toulon, Aix Marseille University, CNRS, IRD, MIO UM 110 La Garde, France (e-mail: guerin@univ-tln.fr).

Color versions of one or more of the figures in this paper are available online at http://ieeexplore.ieee.org.

Digital Object Identifier 10.1109/TGRS.2016.2601242 based on the synthetic aperture radar (SAR) interferometry 40 such as in the Surface Water and Ocean Topography (SWOT) 41 mission concept, Doppler information due to the satellite mo- 42 tion is used to enhance the along-track resolution as it is done 43 in SAR imaging. However, this useful Doppler quantity is 44 affected by a Doppler anomaly due to the motion of waves. The 45 impact of this Doppler anomaly becomes crucial to estimate, 46 as it can impact the SAR ground cells estimated location and 47 azimuthal resolution and, hence, the final geophysical surface 48 estimates. In this respect, the dynamical aspects of the near- 49 nadir backscattered field become crucial, and their impact must 50 be carefully quantified.

There has been a certain number of studies devoted to the 52 analysis of the Doppler signal of the microwave radar echo in 53 the framework of analytic (e.g., [1]-[11]) and rigorous numer- 54 ical scattering models (e.g., [12]-[18]). As shown in some of 55 these works at large and grazing incidence, a relevant descrip- 56 tion of the different mechanisms at the origin of the Doppler 57 spectrum requires a (at least weakly) nonlinear description of 58 water waves combined with advanced scattering models that 59 can take into account complex effects related to polarization, 60 multiple scattering, or shadowing. At low incidence, however, 61 the situation is much simpler since the physical optics (PO) is 62 the reference scattering model, whereas nonlinear interactions 63 at the surface can be neglected in the first place. This opens the 64 way to a complete analytic description of the Doppler spectrum 65 in view of further comparisons with experimental data.

In this paper, we propose a study of the correlation time, as 67 well as the Doppler shift of the backscattered signal induced by 68 wave motion at low incidence. We obtain a simple expression 69 of the decorrelation time with respect to the main oceanic 70 parameters. We further discuss the wave-induced Doppler shift 71 and propose an original approach to calculate the Doppler 72 centroid and the full two-sided Doppler spectrum, which is 73 based on the distribution of the instantaneous Doppler shift. $\quad 74$

\section{Time-Evolving Linear Water Surface}

75

We assume that the elevation of the sea surface about its 76 mean plane is described by a function $z=\eta(\mathbf{r}, t)$ of the hor- 77 izontal coordinate $(\mathbf{r}=(x, y))$. We adopt the classical linear 78 picture in which the time-evolving sea surface can be written as 79 a continuous summation of independent harmonics, i.e.,

$$
\eta(\mathbf{r}, t)=\operatorname{Re}\left\{\int_{\mathbb{R}^{2}} a(\mathbf{k}) e^{i\left(\mathbf{k} \cdot \mathbf{r}-\omega_{\mathbf{k}} t\right)} d \mathbf{k}\right\}
$$


81 where $a(\mathbf{k})$ is the random complex amplitude of the wave 82 associated to wavenumber $\mathbf{k} ; \omega_{k}=\sqrt{g\|\mathbf{k}\|+\gamma_{0}\|\mathbf{k}\|^{3}}$ is the 83 gravity-capillarity wave dispersion relationship with $g=$ $849.81 \mathrm{~m} \cdot \mathrm{s}^{-2}$, i.e., the gravitational constant; and $\gamma_{0}=7.29 \times$ $8510^{-5} \mathrm{~m}^{3} \cdot \mathrm{s}^{-2}$, i.e., the surface tension coefficient of seawater 86 (estimated from [19] with a sea surface temperature of $10{ }^{\circ} \mathrm{C}$ 87 and a salinity of 35 PSU). The spatiotemporal surface corre88 lation function $(\rho(\mathbf{r}, t)=\langle\eta(\mathbf{r}, t) \eta(\mathbf{0}, 0)\rangle$, where $\langle\cdot\rangle$ represents 89 the ensemble average) can be written as

$$
\rho(\mathbf{r}, t)=\operatorname{Re}\left\{\int_{\mathbb{R}^{2}} \Psi(\mathbf{k}) e^{i\left(\mathbf{k} \cdot \mathbf{r}-\omega_{\mathbf{k}} t\right)} d \mathbf{k}\right\}
$$

90 where $\Psi(\mathbf{k})=(1 / 2)\left\langle|a(\mathbf{k})|^{2}\right\rangle$ is the directional wavenumber 91 spectrum. The latter is usually written in polar coordinates $92\left(k, \phi_{k}\right)$ as

$$
\Psi(\mathbf{k})=\frac{\Psi_{0}(k)}{k} F\left(k, \phi_{k}\right)
$$

93 where $\Psi_{0}(k)$ is the omnidirectional spectrum, and $F\left(k, \phi_{k}\right)$ 94 is the spreading function describing the azimuthal variation 95 of wave energy with respect to the wind direction. The di96 rectional spectrum is, in general, not centrosymmetric (i.e., $97 \Psi(-\mathbf{k}) \neq \Psi(\mathbf{k}))$ as waves propagating along or against the 98 main wind direction do not have the same energy. However, 99 the spatial variations of a frozen surface at a given time, for 100 example, $t=0$, are described by a "true" power spectrum, 101 which is the symmetrized version of the directional spectrum, 102 i.e., $\Psi_{s}(\mathbf{k})=(1 / 2)(\Psi(\mathbf{k})+\Psi(-\mathbf{k}))$. The symmetrized spec103 trum $\Psi_{s}$ is relevant for the evaluation of the normalized radar 104 cross section (NRCS) using the classical backscattering model. 105 A popular model is the Elfouhaily et al. unified spectrum [20], 106 whose spreading function is described by a simple biharmonic 107 function, i.e.,

$$
F\left(k, \phi_{k}\right)=\frac{1}{2 \pi}\left\{1+\Delta(k) \cos \left[2\left(\phi_{k}-\phi_{w}\right)\right]\right\}
$$

108 where $0<\Delta(k)<1$ is a contrast function ensuring a correct 109 ratio of upwind/crosswind slopes, and $\phi_{w}$ is the direction of 110 the wind vector with respect to the $x$-axis. However, such 111 directional spectrum, which does not distinguish the upwind 112 and downwind directions, is insufficient to describe the dynam113 ics of waves. Asymmetric spreading functions have been first 114 proposed by Longuet-Higgins et al. [21] and later on refined by 115 Plant [22] in order to preserve the ratio of upwind/crosswind 116 slopes, i.e.,

$$
F\left(k, \phi_{k}\right)=\frac{\left[\cos \left(\frac{\phi_{k}-\phi_{w}}{2}\right)\right]^{2 \gamma(k)}}{\int_{-\pi}^{\pi}\left[\cos \left(\frac{\phi_{k}-\phi_{w}}{2}\right)\right]^{2 \gamma(k)} d \phi_{k}}
$$

117 where

$$
\gamma(k)=-\ln \left(\frac{1-\Delta(k)}{1+\Delta(k)}\right) / \ln 2 .
$$

118 Along this paper, we will adopt this formulation of the spread119 ing function together with the omnidirectional expression of the 120 Elfouhaily et al. spectrum in the numerical experiments.

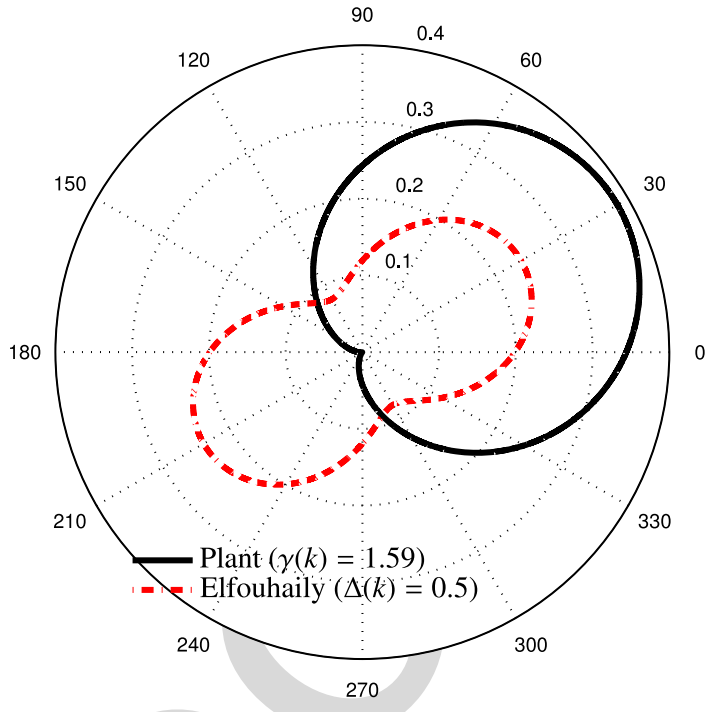

Fig. 1. Comparison of the Elfouhaily 1997 and Plant 2002 spreading functions for $\Delta(k)=0.5 \leftrightarrow \gamma(k)=1.59$. In this example, the wind is oriented at $30^{\circ}$ from the origin. The dashed circles represent the values of the spreading functions $F$.

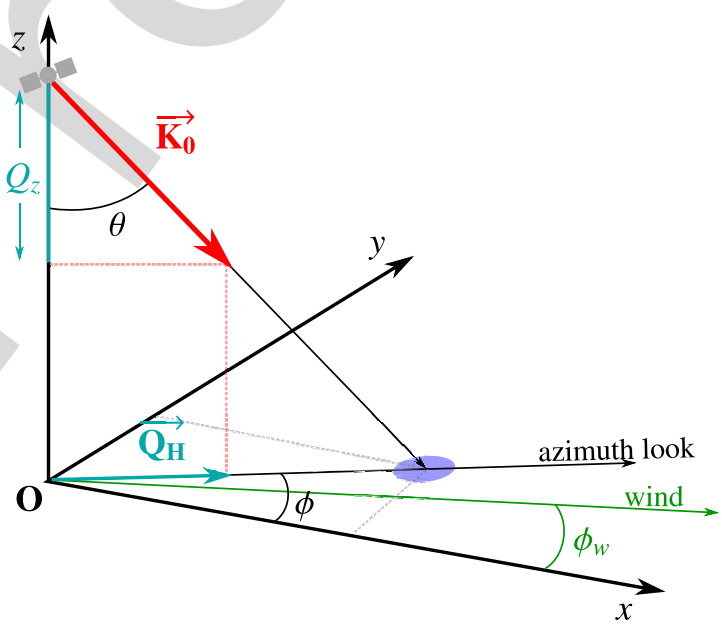

Fig. 2. Schematic of the scattering geometry.

\section{TEMPORAL FIELD CORRELATION}

\section{A. PO Formalism}

As it is customary, we assume the incident field on the 123 sea surface to be a monochromatic plane wave with wave 124 vector $\mathbf{K}_{\mathbf{0}}$ and wavenumber $K_{0}$ (see Fig. 1). The temporal 125 signal recorded on the receiver is proportional to the complex 126 scattering amplitude $\mathbb{S}(t)$ of the backscattered field (see, e.g., 127 [23]). The backscattered amplitude in the PO approximation, 128 which is also known as the Kirchhoff approximation [24], is 129 given by

$$
\mathbb{S}(t)=\frac{1}{(2 \pi)^{2}} \frac{\mathcal{K}}{Q_{z}} \int_{\mathbb{R}^{2}} e^{-i \mathbf{Q}_{\mathbf{H}} \cdot \mathbf{r}} e^{i Q_{z} \eta(\mathbf{r}, t)} d \mathbf{r}
$$

where we have introduced the so-called Ewald vector $\mathbf{Q}=131$ $-2 \mathbf{K}_{\mathbf{0}}$ with its horizontal projection $\mathbf{Q}_{\mathbf{H}}$ and vertical projection 132 $Q_{z}$, and $\mathcal{K}=\mathbf{Q}^{2} \mathcal{R} / 2$ is a geometric kernel, where $\mathcal{R}$ stands for 133 the complex Fresnel reflection coefficient at normal incidence 134 for sea water. Fig. 2 depicts the geometry of the problem. 135 
136 The Cartesian system $(x, y, z)$ is a fixed reference frame. The 137 incidence $(\theta)$ and azimuth $(\phi)$ scattering angles are taken with 138 respect to the $z$ - and $x$-axis, respectively. The azimuthal wind 139 direction is denoted by $\phi_{w}$. In this configuration, the clas140 sical observation directions, namely, upwind, crosswind, and 141 downwind, are obtained for the azimuthal angles $\phi=\phi_{w} \pm \pi$, $142 \phi=\phi_{w} \pm \pi / 2$, and $\phi=\phi_{w}$, respectively. The dependence of 143 the viewing angles in expression (7) is implicit. Under the 144 assumption of a Gaussian random process for the sea surface, 145 the resulting field correlation function is given by

$$
\begin{aligned}
C_{\mathrm{PO}}(t) & =\lim _{A \rightarrow \infty} \frac{4 \pi}{A}\left(\left\langle\mathbb{S}(t) \mathbb{S}^{*}(0)\right\rangle-|\langle\mathbb{S}(0)\rangle|^{2}\right) \\
& =\frac{1}{\pi} \frac{|\mathcal{K}|^{2}}{Q_{z}^{2}} \int_{\mathbb{R}^{2}} e^{-i \mathbf{Q}_{\mathbf{H}} \cdot \mathbf{r}}\left[e^{-Q_{z}^{2}\left[\rho_{0}-\rho(\mathbf{r}, t)\right]}-e^{-Q_{z}^{2} \rho_{0}}\right] d \mathbf{r}
\end{aligned}
$$

146 where $\rho_{0}$ denotes the surface correlation at the origin (in time 147 and space). Note that the field correlation at the origin, i.e., $148 C_{\mathrm{PO}}(0)$, reduces to the well-known expression of the NRCS in 149 the Kirchhoff approximation, i.e., $\sigma_{\mathrm{PO}}^{0}$. However, the numerical 150 evaluation of this correlation is, in general, more difficult than 151 the ordinary NRCS and deserves a specific numerical procedure 152 using polar coordinates and azimuthal Fourier series expansion.

\section{B. GO Formalism}

154 A well-known approximation of the PO, which is valid in 155 the limit of short radar wavelength, is the geometrical optics 156 (GO) approximation. Simple algebra using a spatiotemporal 157 second-order Taylor expansion of the surface autocorrelation 158 function $\rho$ in the integrand (8) leads to the following expression 159 for the field correlation function in the GO approximation 160 (see the Appendix for detailed calculations):

$$
C_{\mathrm{GO}}(t)=\sigma_{\mathrm{GO}}^{0} \times T_{\mathrm{GO}}(t) \times \mathbb{M}_{\mathrm{GO}}(t) .
$$

161 Here, $\sigma_{\mathrm{GO}}^{0}$ is the classical expression of the NRCS in the GO 162 approximation, i.e.,

$$
\begin{aligned}
\sigma_{\mathrm{GO}}^{0}=\frac{|\mathcal{R}|^{2}}{2 \Sigma \cos ^{4} \theta} \exp & \left(-\frac{\tan ^{2} \theta}{2 \Sigma^{2}}\left(s_{y y}^{2} \cos ^{2} \phi\right.\right. \\
& \left.\left.-2 s_{x y}^{2} \sin \phi \cos \phi+s_{x x}^{2} \sin ^{2} \phi\right)\right)
\end{aligned}
$$

163 with the directional mean-square slopes (the derivatives are 164 taken at the origin)

$$
\begin{aligned}
s_{x x}^{2} & =-\frac{\partial^{2} \rho}{\partial x^{2}}, s_{y y}^{2}=-\frac{\partial^{2} \rho}{\partial y^{2}}, s_{x y}^{2}=-\frac{\partial^{2} \rho}{\partial x \partial_{y}} \\
\Sigma^{2} & =s_{x x}^{2} s_{y y}^{2}-s_{x y}^{4} .
\end{aligned}
$$

165 The second term $T_{\mathrm{GO}}$ is a Gaussian damping function, i.e.,

$$
T_{\mathrm{GO}}(t)=\exp \left(-2 K_{0}^{2} L^{2} \cos ^{2} \theta t^{2}\right)
$$

166 with

$$
\begin{aligned}
L^{2} & =s_{t t}^{2}-\frac{s_{y y}^{2} s_{x t}^{2}-2 s_{x y}^{2} s_{x t} s_{y t}+s_{x x}^{2} s_{y t}^{2}}{\Sigma^{2}} \\
s_{t t}^{2} & =-\frac{\partial^{2} \rho}{\partial t^{2}}, s_{x t}=\frac{\partial^{2} \rho}{\partial x \partial t}, s_{y t}=\frac{\partial^{2} \rho}{\partial y \partial t} .
\end{aligned}
$$

The second-order time derivative $s_{t t}^{2}$ can be interpreted as the 167 variance of the vertical orbital velocity of waves. The spa- 168 tiotemporal cross-derivatives $s_{x t}$ and $s_{y t}$ have no such simple 169 interpretation. The third term in (9) is a complex azimuthal 170 modulation function, i.e.,

$$
\begin{aligned}
& \mathbb{M}_{\mathrm{GO}}(t)=\exp \left(-i \frac{2 K_{0} t}{\Sigma^{2}} \sin \theta\right. \\
& \left.\times\left[\left(s_{y y}^{2} s_{x t}-s_{x y}^{2} s_{y t}\right) \cos \phi+\left(s_{x x}^{2} s_{y t}-s_{x y}^{2} s_{x t}\right) \sin \phi\right]\right) .
\end{aligned}
$$

Note that the expression of the temporal correlation in the GO 172 framework solely depends on the spatiotemporal second-order 173 derivatives of the surface correlation function at the origin. 174 These coefficients can be easily obtained using the correspond- 175 ing moments of the power spectrum.

\section{Correlation Time}

The correlation time $\tau_{c}$ of the backscattered field is typically 178 defined by

$$
\left|C\left(\tau_{c}\right)\right|=a C(0)
$$

where $|\cdot|$ stands for the absolute value (modulus), for some 180 threshold $0<a<1$. This quantity must be numerically evalu- 181 ated in the PO formalism but can be analytically estimated in 182 the GO formalism, with $T_{\mathrm{GO}}\left(\tau_{c}\right)=a$, yielding

$$
\tau_{c}=\frac{\sqrt{-\ln (a) / 2}}{K_{0}|\cos \theta| L} .
$$

Note that the correlation time does not depend on the azimuth 184 angle in the GO formalism, which is confirmed by numerically 185 calculating this correlation time with the PO formalism. More- 186 over, we numerically found that the correlation times calculated 187 from the PO formalism and from the GO formalism are very 188 close in $\mathrm{Ku}$ - and Ka-bands, although the corresponding NRCS 189 can be significantly different (i.e., the GO formalism is not 190 valid to evaluate the NRCS at low incidence but useful to 191 evaluate the correlation time). At an incidence angle of $\theta=20^{\circ}, 192$ which is in the limit of validity of the models, we found an 193 absolute difference smaller than $0.1 \mathrm{~ms}$ between the PO and 194 GO correlation times.

The simple analytic expression (18) of the correlation time 196 shows that the field decorrelation is mainly due to the motion 197 of the wave field in the line-of-sight of the radar through the 198 vertical orbital wave velocity $\left(s_{t t}^{2}\right)$, which is numerically found 199 to be the dominant term in (14), i.e., $L \simeq s_{t t}$. This quantity is 200 related to the first moment of the wave spectrum and is mainly 201 contributed to by long waves, i.e.,

$$
s_{t t}^{2}=\int_{0}^{\infty} \omega_{k}^{2} \Psi_{0}(k) d k \simeq g \int_{0}^{\infty} k \Psi_{0}(k) d k .
$$

For wind waves spectra in the gravity range, i.e., $\Psi_{0}(k) \sim k^{-3} 203$ and $s_{t t}^{2} \simeq g k_{p} H_{s}^{2} / 8$, where $k_{p}$ is the peak wavenumber. Hence, 204 


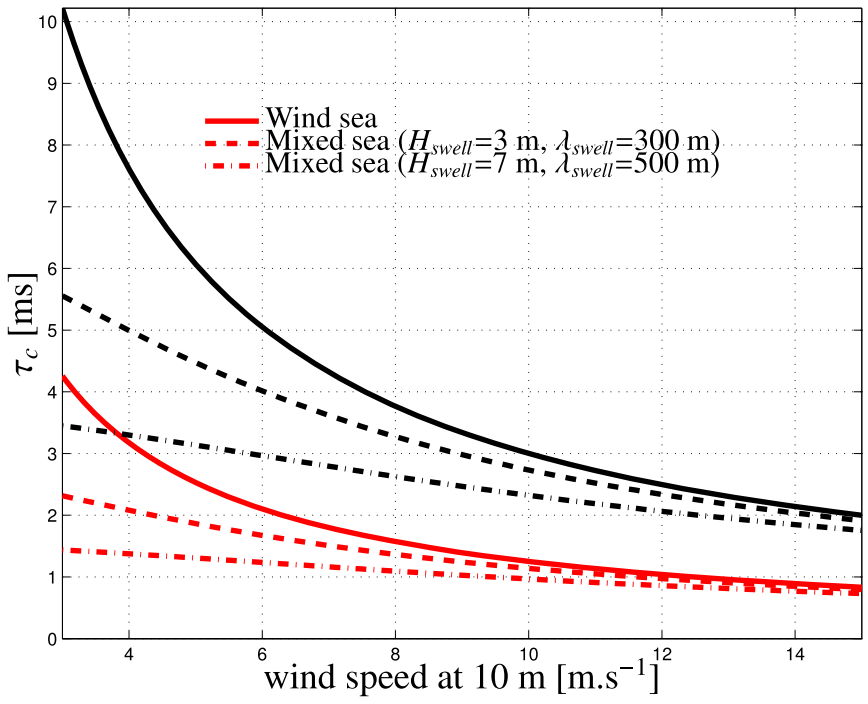

Fig. 3. Correlation time $\tau_{c}$ in (red lines) Ka-band $\left(f_{0}=36 \mathrm{GHz}\right)$ and (black lines) Ku-band $\left(f_{0}=15 \mathrm{GHz}\right)$ at nadir as a function of wind speed at $10 \mathrm{~m}$ for the decorrelation threshold $a=1 / e$.

205 the correlation time can be simply expressed in terms of the 206 main oceanic parameters $k_{p}$ and $H_{s}$, i.e.,

$$
\tau_{c} \simeq \frac{2 \sqrt{-\ln (a)}}{K_{0}|\cos \theta| \sqrt{g k_{p}} H_{s}} .
$$

207 In the case of a mixed sea composed of a swell (with wavenum208 ber $k_{\text {swell }}$ and significant wave height $\left.H_{\text {swell }}\right)$ and a wind sea 209 (with peak wavenumber $k_{p}$ and significant wave height $H_{\text {wind }}$, 210 the aforementioned formula can be easily adapted with $s_{t t}^{2} \simeq$ $211 g k_{p} H_{\text {wind }}^{2} / 8+g k_{\text {swell }} H_{\text {swell }}^{2} / 16$.

212 Fig. 3 shows a comparison of the correlation time $\tau_{c}$ in $\mathrm{Ku}-$ $213(15 \mathrm{GHz})$ and $\mathrm{Ka}$-band $(36 \mathrm{GHz})$ at nadir as a function of 214 wind speed at $10 \mathrm{~m}$ above the sea surface, with a decorrelation 215 threshold set to $a=1 / e$. It has been calculated with both the 216 GO-based formula (18) and the PO model, with no numerical 217 difference. In addition to a pure wind sea case, a mixed sea 218 with a swell of wavelength longer than the dominant wind 219 sea wavelength (for a $15 \mathrm{~m} \cdot \mathrm{s}^{-1}$ wind speed, the dominant 220 wavelength is about $200 \mathrm{~m}$ ) has been considered. For a pure 221 wind sea, the correlation time is decreased by a factor 4-5 222 from low to high wind speed. In the case of a mixed sea, the 223 correlation time is less sensitive to the wind speed and is mainly 224 driven by the swell parameters. It ranges from 0.5 to $4 \mathrm{~ms}$ in $225 \mathrm{Ka}$-band and from 2 to $10 \mathrm{~ms}$ in $\mathrm{Ku}$-band. The simplified 226 expression (20) for the correlation time has been compared with 227 expression (18) and is found in excellent agreement (with at 228 most a few percent relative difference), showing that the corre229 lation time can be expressed in a simple and accurate manner 230 with only the peak wavenumber and the significant wave height. 231 A study of the phase of the backscattered signal from the sea 232 surface has been conducted by Chapman et al. [25], where au233 thors give measurements of the backscattered signal correlation 234 time at nadir for different microwave frequencies. Results are 235 given for a developed sea with $u_{10} \simeq 4.7 \mathrm{~m} / \mathrm{s}$ with negligible 236 swell. Using the classical relation for fully developed wind 237 seas, i.e., $H_{s}=0.025 \times u_{10}^{2}$ and $k_{p}=0.84^{2} \mathrm{~g} / u_{10}^{2}$, we obtain

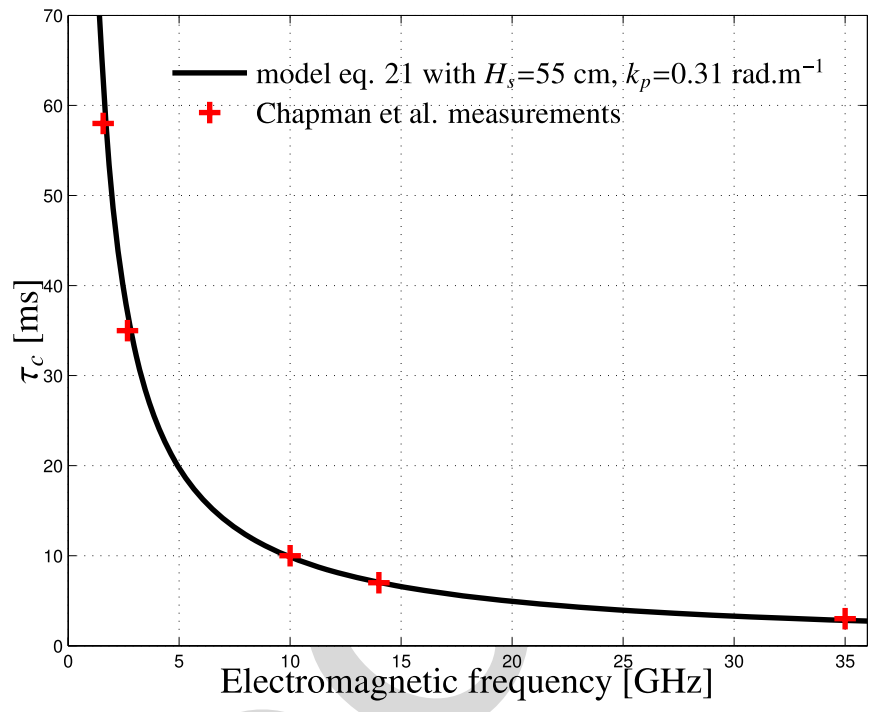

Fig. 4. Comparison of the correlation time according to model (20) and Chapman et al. [25] measurements as a function of the electromagnetic frequency at nadir for a decorrelation threshold $a=1 / \mathrm{e}$.

$k_{p} \simeq 0.31 \mathrm{rad} \cdot \mathrm{m}^{-1}$ and $H_{s} \simeq 55 \mathrm{~cm}$. Fig. 4 compares the 238 measurements of the decorrelation time by Chapman et al. 239 and the estimation after formula (20) as a function of radar 240 frequency. An excellent agreement is obtained.

\section{WAVE-INDUCED DOPPLER SHIFT}

\section{A. Different Approaches to the Doppler Centroid}

As it is well known, the backscattered time signal undergoes 244 a Doppler shift due to the combined motion of the platform 245 (airborne or spaceborne sensor) and the motion of waves at the 246 sea surface. The dominant contribution to the Doppler centroid 247 frequency arises from the relative velocity of the platform with 248 respect to the ground. It can be easily estimated and removed 249 by means of a geometrical model. The residual Doppler shift, 250 or Doppler anomaly, due to wave motion is much smaller but 251 induces azimuthal smearing resulting in the loss of resolution 252 in the SAR image. Here, we will try to estimate its statis- 253 tical properties as a function of sea state. There are several 254 approaches to calculate the Doppler shift of waves. The most 255 classical technique is the frequency-domain estimation (FDE) 256 based on the Doppler spectrum. For this, the Fourier transform 257 of the temporal correlation function is taken, i.e.,

258

$$
D(f)=\int_{-\infty}^{+\infty} e^{-2 i \pi f t} C(t) d t
$$

and the Doppler shift is defined as the mean (e.g., [7] and [8]) or 259 median (see [26]) frequency with respect to the normalized dis- 260 tribution $D(f) / \int D(f) d f$. In practice, the Doppler spectrum 261 $D(f)$ is obtained through the variance of the periodogram of 262 the time signal (i.e., the sample average of $|\operatorname{FFT}(\mathbb{S}(t))|^{2}$ ). The 263 advantage of the technique is that it gives the full shape of 264 the Doppler spectrum and discriminate positive and negative 265 frequency shifts $(D(-f) \neq D(f)$, in general). Note that the 266 estimation of the Doppler shift based on the mean or median of 267 
268 the Doppler spectrum is meaningful only if the latter is essen269 tially half-sided, as the negative and frequency components of 270 the two-sided spectrum would cancel out and make the mean 271 Doppler shift actually close to zero. The difficulty of the 272 technique for simulation purposes is the full calculation of the 273 temporal correlation function necessary to estimate the Doppler 274 spectrum. To avoid this calculation, one often limits oneself 275 to the estimation of the first two moments of the Doppler 276 spectrum, which can be obtained with the time derivative of the 277 signal correlation function at the origin (see, e.g., [7] and [8]).

278 Another approach is the time-domain estimator (TDE), 279 which has been found advantageous in the context of SAR 280 systems (see [26]). It consists in estimating the phase of the 281 complex signal correlation, i.e.,

$$
C(t)=|C(t)| e^{i 2 \pi t f_{c}} .
$$

282 The mean Doppler shift frequency is then simply obtained with

$$
f_{c}=\frac{1}{t} \arg (C(t)) \text {. }
$$

283 This approach (also employed in [6] in the context of analytic 284 scattering models) has the merits of simplicity but does not 285 provide the dispersion around the Doppler shift.

286 An alternative TDE can be obtained using the instantaneous 287 random signal, $\mathbb{S}(t)$ instead of its correlation function. We 288 define the instantaneous Doppler frequency shift induced by 289 wave motion as

$$
f(t)=-\frac{1}{2 \pi} \partial_{t} \varphi(t)
$$

290 where $\varphi(t)$ is the scattering phase of the illuminated target (we 291 note that with this convention, positive frequencies represent 292 waves traveling to the radar). The scattering phase is related to 293 the complex backscattered signal $\mathbb{S}(t)$ through

$$
\varphi(t)=\arg (\mathbb{S}(t))=\arctan \left[\frac{\operatorname{Im}(\mathbb{S}(t))}{\operatorname{Re}(\mathbb{S}(t))}\right]
$$

294 from which we can infer the simple expression

$$
f(t)=-\frac{1}{2 \pi} \operatorname{Im}\left[\frac{\partial_{t} \mathbb{S}(t)}{\mathbb{S}(t)}\right] .
$$

295 We define the instantaneous Doppler shift distribution as 296 the probability density function (pdf) associated to this last 297 quantity.

\section{B. Doppler Shift in the PO Formalism}

299 Using expression (7) of the scattered field in the PO approx300 imation, we obtain

$$
f(t)=-\frac{1}{2 \pi} \operatorname{Im}\left(\frac{N_{t}}{D_{t}}\right)
$$

301 with

$$
\left\{\begin{array}{l}
N_{t}=i Q_{z} \int_{\mathbb{R}^{2}} \partial_{t} \eta(\mathbf{r}, t) e^{-i \mathbf{Q}_{\mathbf{H}} \cdot \mathbf{r}} e^{i Q_{z} \eta(\mathbf{r}, t)} d \mathbf{r} \\
D_{t}=\int_{\mathbb{R}^{2}} e^{-i \mathbf{Q}_{\mathbf{H}} \cdot \mathbf{r}} e^{i Q_{z} \eta(\mathbf{r}, t)} d \mathbf{r}
\end{array}\right.
$$

Here, a common normalization factor $2 \pi \sqrt{A}$ appeared in the 302 calculation and vanished to make these quantities indepen- 303 dent of the illuminated area $A$. By virtue of the central limit 304 theorem, these random surface integrals follow a centered 305 complex-normal distribution. Classical two-point calculations 306 on Gaussian random variables lead to the following expressions 307 for their respective co- and cross-variances:

$$
\left\{\begin{aligned}
\left\langle\left|N_{t}\right|^{2}\right\rangle= & -Q_{z}^{2} \int_{\mathbb{R}^{2}}\left[Q_{z}^{2}\left(\partial_{t} \rho(\mathbf{r}, 0)\right)^{2}+\partial_{t t} \rho(\mathbf{r}, 0)\right] \\
& \times e^{-i \mathbf{Q}_{\mathbf{H}} \cdot \mathbf{r}} e^{-Q_{z}^{2}\left[\rho_{0}-\rho(\mathbf{r}, 0)\right]} d \mathbf{r} \\
\left\langle\left|D_{t}\right|^{2}\right\rangle= & \int_{\mathbb{R}^{2}} e^{-i \mathbf{Q}_{\mathbf{H}} \cdot \mathbf{r}} e^{-Q_{z}^{2}\left[\rho_{0}-\rho(\mathbf{r}, 0)\right]} d \mathbf{r} \\
\left\langle N_{t}^{*} D_{t}\right\rangle= & Q_{z}^{2} \int_{\mathbb{R}^{2}} \partial_{t} \rho(\mathbf{r}, 0) e^{-i \mathbf{Q}_{\mathbf{H}} \cdot \mathbf{r}} e^{-Q_{z}^{2}\left[\rho_{0}-\rho(\mathbf{r}, 0)\right]} d \mathbf{r} .
\end{aligned}\right.
$$

Note that these quantities are time independent, with the second 309 term equal to the NRCS in PO approximation, apart from a 310 geometrical factor. The pdf associated to the phase derivative 311 (27) can be obtained using recent results on the ratio of two 312 correlated complex-Gaussian random variables (see [27]). It 313 can be expressed as a nonstandardized Student's $t$-distribution 314

$$
p(f)=\frac{\pi}{\sqrt{2} \Delta f}\left[1+\frac{1}{2}\left(\frac{f-f_{c}}{\Delta f}\right)^{2}\right]^{-3 / 2}
$$

with the mean (central) Doppler shift frequency

$$
f_{c}=\frac{C_{i}}{2 \pi} \frac{\left\langle\left|N_{t}\right|^{2}\right\rangle^{\frac{1}{2}}}{\left\langle\left|D_{t}\right|^{2}\right\rangle^{\frac{1}{2}}}
$$

and the dispersion parameter

$$
(\Delta f)^{2}=\frac{1-|C|^{2}}{8 \pi^{2}} \frac{\left\langle\left|N_{t}\right|^{2}\right\rangle}{\left\langle\left|D_{t}\right|^{2}\right\rangle} .
$$

It involves the complex cross correlation of the random vari- 317 ables $N_{t}$ and $D_{t}$, i.e.,

$$
C=\frac{\left\langle N_{t}^{*} D_{t}\right\rangle}{\sqrt{\left\langle\left|N_{t}\right|^{2}\right\rangle\left\langle\left|D_{t}\right|^{2}\right\rangle}}
$$

which is decomposed into real and imaginary parts, i.e., $C=319$ $C_{r}+i C_{i}$. From this, we obtain a simple representation of 320 the Doppler shift distribution after (24) requiring only the 321 calculation of the three surface integrals in (29) involved in 322 the three statistical parameters (31)-(33). Note that expression 323 (31) of the mean Doppler shift is consistent with expression 324 [7, eq. (IV.12)] derived from the first moment of the Doppler 325 spectrum obtained with the FDE. Note, however, that the 326 Doppler shift distribution (30) does not possess a finite variance, 327 contrarily to the latter definition. Nevertheless, the nonstandard- 328 ized Student's $t$-distribution possesses a dispersion parameter 329 $(\Delta f)$ allowing to characterize the dispersion around its mean. 330 We performed a numerical calculation of the mean Doppler 331 shift due to wave motion $\left(f_{c}\right)$ and its dispersion $(\Delta f)$ with 332 the sea spectrum described in Section II. An efficient numerical 333 evaluation of the surface integrals in (29) has been achieved 334 using an integration in polar coordinates together with an az- 335 imuthal Fourier expansion of the surface correlation functions 336 


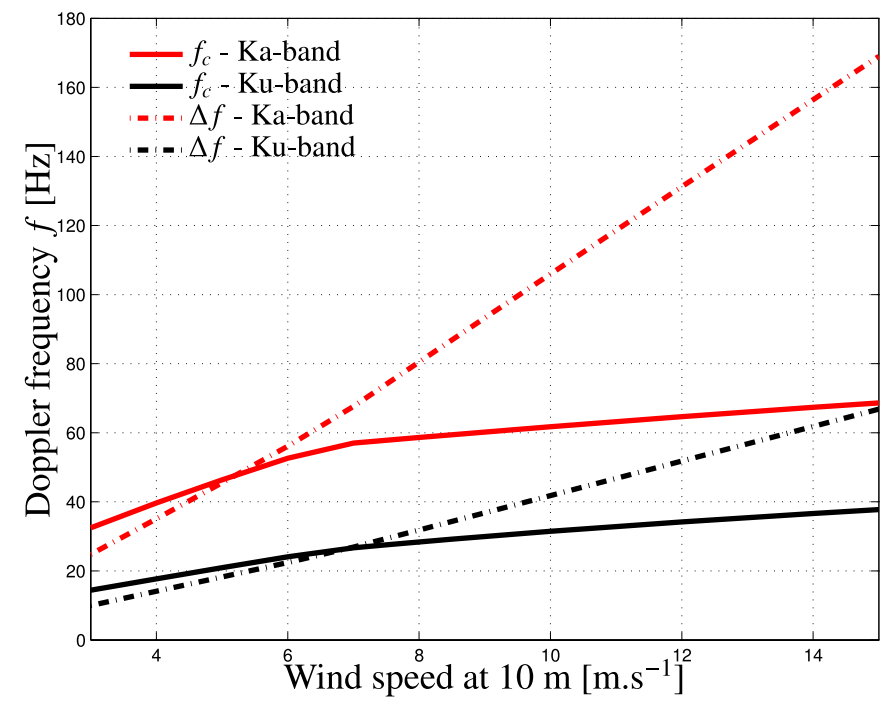

Fig. 5. Evolution of the residual Doppler shift parameters: central frequency $f_{c}$ (plain) and dispersion parameter $\Delta_{f}$ (dash-dotted) as a function of surface roughness represented with the wind speed at $10 \mathrm{~m}$ for an incidence angle $\theta=5^{\circ}$ in the upwind direction. Red plots represent the calculation in Ka-band $\left(f_{0}=36 \mathrm{GHz}\right)$, and black plots represent the calculation in $\mathrm{Ku}$-band $\left(f_{0}=\right.$ $15 \mathrm{GHz})$.

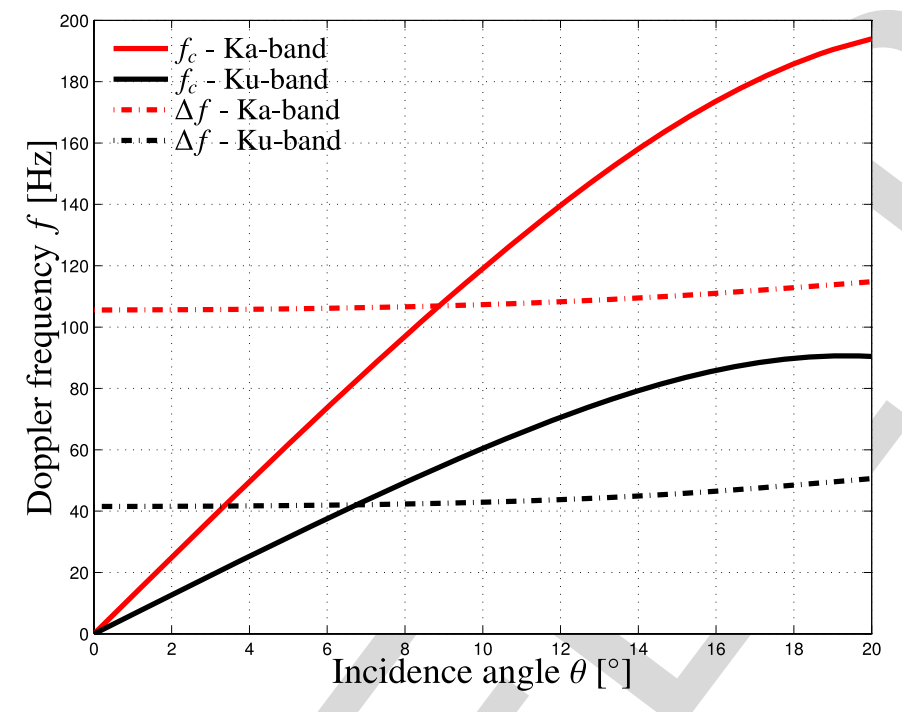

Fig. 6. Same as Fig. 5 except that parameters are plotted as a function of the incidence angle $\theta$ for a wind speed at $10 \mathrm{~m}$ of $10 \mathrm{~m} \cdot \mathrm{s}^{-1}$ in the upwind direction.

337 and related quantities. Figs. 5-7 describe the evolution of the 338 center and width of the Doppler shift distribution as a function 339 of surface roughness (see Fig. 5), incidence angle $\theta$ (see Fig. 6), 340 and azimuth angle $\phi$ (see Fig. 7) for the two microwave bands, 341 i.e., $\mathrm{Ka}\left(f_{0}=36 \mathrm{GHz}\right)$ and $\mathrm{Ku}\left(f_{0}=15 \mathrm{GHz}\right)$.

342 The mean and dispersion parameters of the residual Doppler 343 shift have a similar behavior in the two microwave bands, 344 except that absolute values are higher in Ka-band. In our 345 calculations, their ratio between the two bands is found to 346 be nearly constant and about 2 for the central frequency and 3472.5 for the dispersion parameter regardless of the scattering 348 geometry and surface roughness. Note that the ratio of the 349 residual Doppler shift central frequencies is close but not equal 350 to the ratio of radar wavelengths (2.4). Fig. 5 shows that the

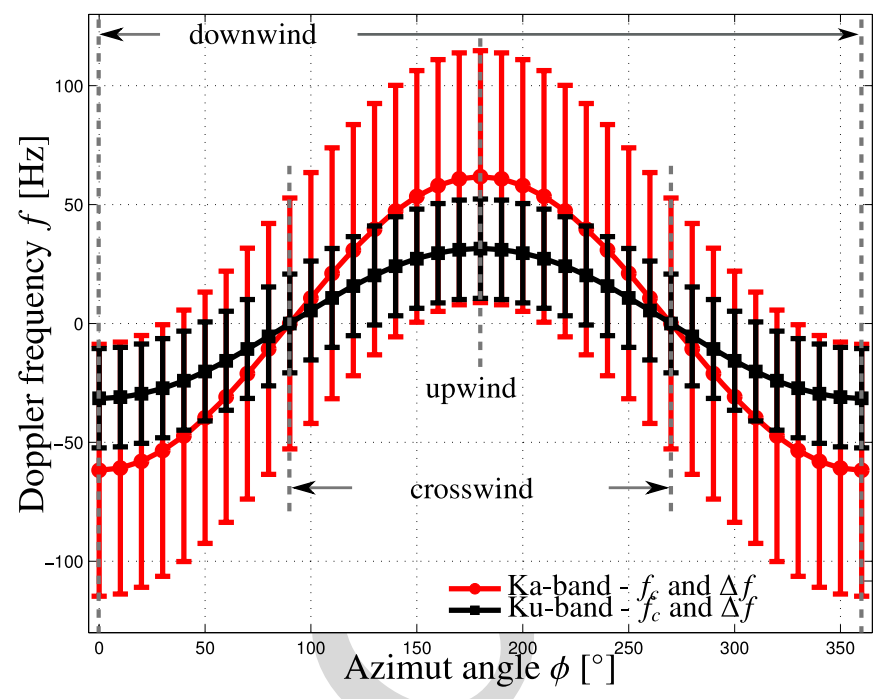

Fig. 7. Same as Fig. 5 except that parameters are plotted as a function of the azimuth angle $\phi$ for an incidence angle $\theta=5^{\circ}$, a wind speed at $10 \mathrm{~m}$ of $10 \mathrm{~m} \cdot \mathrm{s}^{-1}$, and a wind direction $\phi_{w}=0^{\circ}$. Dispersion parameter $\Delta_{f}$ is plotted as an error bar around its mean $f_{c}$.

central frequency $f_{c}$ has a weak sensitivity to sea state, whereas 351 the dispersion parameter undergoes a dramatic increase with 352 wind speed. Hence, it seems that the width of the Doppler 353 shift distribution is a better proxy for wind speed estimation 354 than its mean value. The situation is opposite when it comes 355 to the dependence on the scattering angles: Fig. 6 shows that 356 central frequency $f_{c}$ quickly increases with the incidence angle, 357 whereas the dispersion parameter remains quite constant. The 358 same qualitative behavior holds for the azimuthal dependence, 359 with a central frequency oscillating between positive (upwind 360 angular sector) and negative (downwind angular sector) values 361 and a quasi-constant dispersion parameter. A noticeable result 362 is that the central frequency follows a sinusoidal dependence 363 on the azimuthal angle as will be confirmed later on the basis of 364 theoretical considerations (see the discussion in Section VI-A). 365

\section{TWo-Sided Doppler SPECTRUM}

Doppler spectra in the microwave regime exhibit, in general, 367 asymmetric components in the positive and negative frequen- 368 cies related to the velocities of waves traveling to and away 369 from the radar. The previous definition of the instantaneous fre- 370 quency (27) does not allow to differentiate the progressive and 371 regressive parts of the surface, which separately contributes to 372 the two parts of the Doppler spectrum. Hence, the instantaneous 373 Doppler shift distribution (30) is expected to be consistent 374 with normalized Doppler spectra, according to the classical 375 definition (21) for the one-sided spectra only (i.e., if all waves 376 are supposed to travel in the same direction). To be able to 377 distinguish positive and negative frequencies, we mathemat- 378 ically decompose the surface into a sum of progressive and 379 regressive waves (i.e., waves traveling to or against the radar 380 look direction)

$$
\eta(\mathbf{r}, t)=\eta^{+}(\mathbf{r}, t)+\eta^{-}(\mathbf{r}, t)
$$

where $\eta^{+}$(respectively, $\eta^{-}$) is defined by the integral (1), with 382 a domain of integration restricted to wave vectors in the same 383 

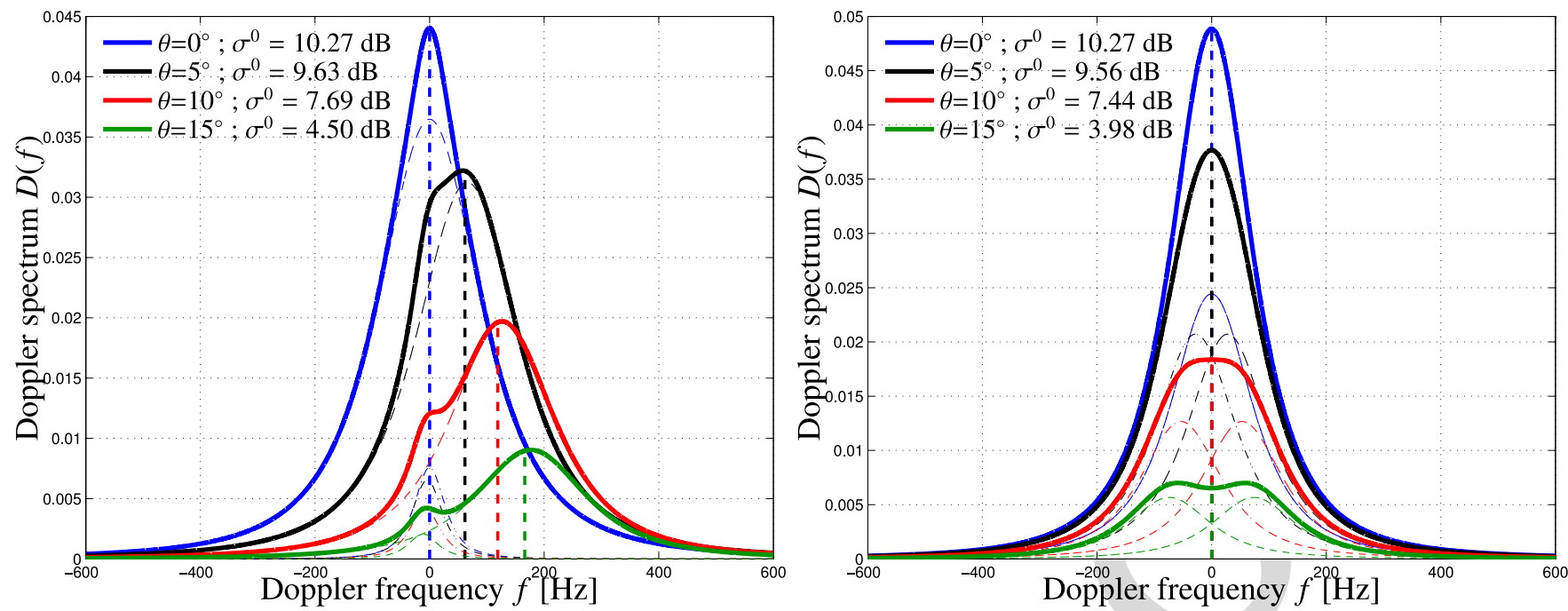

Fig. 8. Examples of the recomposed Doppler spectrum (thick lines) calculated for a wind speed at $10 \mathrm{~m} \mathrm{of} 10 \mathrm{~m} \cdot \mathrm{s}^{-1}$ in $\mathrm{Ka}-\mathrm{band}\left(f_{0}=36 \mathrm{GHz}\right)$ and several values of incidence angles $\theta$. (Left) Calculation is made in the upwind direction. (Right) Calculation is made in the crosswind direction. The thin dashed lines represent the progressive part, and the thin dash-dotted lines the regressive part of the Doppler spectrum. The thick dashed lines represent the central frequency of the Doppler shift $f_{c}$.

384 half-plane (respectively, opposite half-plane) as the radar inci385 dent wavenumber, i.e., $\mathbf{K}_{\mathbf{0}} \cdot \mathbf{k}>0$ (respectively, $\mathbf{K}_{\mathbf{0}} \cdot \mathbf{k}<0$ ). 386 We accordingly decompose the surface autocorrelation function 387 with

$$
\rho(\mathbf{r}, t)=\rho^{+}(\mathbf{r}, t)+\rho^{-}(\mathbf{r}, t)
$$

388 where, analogously, $\rho^{ \pm}$is defined through the spectral integral 389 (2) restricted to the integration domain $\pm \mathbf{K}_{\mathbf{0}} \cdot \mathbf{k}>0$. We now 390 assume that the Doppler spectrum is the summation of two 391 subspectra obtained by assuming that only one category of 392 waves is moving (progressive or regressive), whereas the other 393 is frozen. Each subspectrum is proportional to the distribution $394 p^{ \pm}$of instantaneous Doppler shifts $f^{ \pm}$associated to the pro395 gressive and regressive parts of the surface, i.e.,

$$
f^{ \pm}(t)=-\frac{1}{2 \pi} \partial_{t} \varphi^{ \pm}(t)=-\frac{1}{2 \pi} \operatorname{Im}\left[\frac{\partial_{t} \mathbb{S}^{ \pm}(t)}{\mathbb{S}(t)}\right]
$$

396 where it is understood that the time derivation $\partial_{t} \mathbb{S}^{+}$is taken 397 with respect to the progressive part of the surface only $\left(\eta^{+}\right)$, 398 the regressive part $\left(\eta^{-}\right)$being frozen, and conversely for $\partial_{t} \mathbb{S}^{-}$. 399 We can therefore rewrite

$$
D(f)=\alpha^{+} p^{+}(f)+\alpha^{-} p^{-}(f)
$$

AQ5 400 for some weights $\alpha^{ \pm}$to be determined. The moments on the 401 order of 0 and 1 of the Doppler spectrum must satisfy the fol402 lowing consistency relation with the NRCS $\left(\sigma^{0}\right)$ and the mean 403 Doppler shift:

$$
\left\{\begin{array}{l}
\sigma^{0}=\alpha^{+}+\alpha^{-} \\
\sigma^{0} f_{c}=\alpha^{+} f_{c}^{+}+\alpha^{-} f_{c}^{-}
\end{array}\right.
$$

404 where $f_{c}$ and $f_{c}^{ \pm}$are the mean frequencies associated to the 405 Doppler spectrum and its subspectra

$$
f_{c}=\int_{\mathbb{R}} f p(f) d f, f_{c}^{ \pm}=\int_{\mathbb{R}} f p^{ \pm}(f) d f .
$$

By solving this last system of equations, we obtain

$$
\alpha^{ \pm}= \pm \sigma^{0} \frac{f_{c}-f_{c}^{\mp}}{f_{c}^{+}-f_{c}^{-}} \text {. }
$$

The calculation of the pdf of the phase derivatives $\left(p^{ \pm}\right)$in 407 the PO formalism is very similar to the calculation developed in 408 Section IV-B, with the difference that the time derivation should 409 be taken with respect to progressive or regressive waves only. 410 The formula (28)-(33) remain similar with the only change that 411 $N_{t}$ and related quantities should be replaced by

$$
\left\{\begin{aligned}
& N_{t}^{ \pm}=i Q_{z} \int_{\mathbb{R}^{2}} \partial_{t} \eta^{ \pm}(\mathbf{r}, t) e^{-i \mathbf{Q}_{\mathbf{H}} \cdot \mathbf{r}} e^{i Q_{z} \eta(\mathbf{r}, t)} d \mathbf{r} \\
&\left\langle\left|N_{t}^{ \pm}\right|^{2}\right\rangle=-Q_{z}^{2} \int_{\mathbb{R}^{2}}\left[Q_{z}^{2}\left(\partial_{t} \rho^{ \pm}(\mathbf{r}, 0)\right)^{2}+\partial_{t t} \rho^{ \pm}(\mathbf{r}, 0)\right] \\
& \times e^{-i \mathbf{Q} \mathbf{Q}_{\mathbf{H}} \cdot \mathbf{r}} e^{-Q_{z}^{2}\left[\rho_{0}-\rho(\mathbf{r}, 0)\right]} d \mathbf{r} \\
&\left\langle N_{t}^{ \pm *} D_{t}\right\rangle= Q_{z}^{2} \int_{\mathbb{R}^{2}} \partial_{t} \rho^{ \pm}(\mathbf{r}, 0) e^{-i \mathbf{Q}_{\mathbf{H}} \cdot \mathbf{r}} e^{-Q_{z}^{2}\left[\rho_{0}-\rho(\mathbf{r}, 0)\right]} d \mathbf{r}
\end{aligned}\right.
$$

Fig. 8 shows an example of the two-sided Doppler spectrum 413 in Ka-band at $10 \mathrm{~m} / \mathrm{s}$ wind speed for different incidence angles. 414 At low incidence, the positive and negative Doppler spectra 415 merged into a single wider peak.

\section{Estimation of Surface Parameters}

\section{A. Wind Direction and Doppler Shift}

418

Recent studies with coherent radars have established a clear 419 relationship between the centroid of the Doppler anomaly 420 and the wind vector above the sea surface (e.g., [28]-[31]) 421 at moderate and large incidence angles $\left(>20^{\circ}\right)$. However, at 422 low incidence, the central residual Doppler shift has a weak 423 dynamic with respect to wind speed and is smaller than the 424 dispersion parameter, as shown in Fig. 5. This makes the 425 Doppler anomaly, when taken at fixed angles, a bad tracer for 426 the wind vector. Nevertheless, a clear dependence with wind 427 direction can be seen on the azimuthal variations of the Doppler 428 shift central frequency. The sinusoidal variation observed in 429 Fig. 7 can be well understood using GO-like developments 430 
431 for the central frequency (31) expressed in the PO formalism. 432 The main advantage of this formulation is that the central 433 frequency is expressed as a function of statistical parameters of 434 the surface and does not depend on the chosen wave spectrum. 435 The expression of the central frequency is the same as the 436 complex azimuthal modulation function of the GO formalism 437 (16) and is expressed, in term of central frequency, i.e.,

$$
\begin{aligned}
f_{c}=-\frac{K_{0} \sin \theta}{\pi \Sigma^{2}}\left[\left(s_{y y}^{2} s_{x t}-\right.\right. & \left.s_{x y}^{2} s_{y t}\right) \cos \phi \\
& \left.+\left(s_{x x}^{2} s_{y t}-s_{x y}^{2} s_{x t}\right) \sin \phi\right]
\end{aligned}
$$

438 which can be rewritten as

$$
f_{c}=-F_{c} \cos \left(\phi-\phi_{w}\right)
$$

439 where

$$
F_{c}=\frac{K_{0} \sin \theta}{\pi \Sigma^{2}} \sqrt{\left(s_{y y}^{2} s_{x t}-s_{x y}^{2} s_{y t}\right)^{2}+\left(s_{x x}^{2} s_{y t}-s_{x y}^{2} s_{x t}\right)^{2}}
$$

440 is the maximum central frequency, and

$$
\tan \left(\phi_{w}\right)=\frac{s_{x x}^{2} s_{y t}-s_{x y}^{2} s_{x t}}{s_{y y}^{2} s_{x t}-s_{x y}^{2} s_{y t}}
$$

441 is the wind direction. The approximate formulas (42)-(45) 442 based on GO-like expansion therefore explains the observed 443 sinusoidal variation with the azimuthal angle observed in the 444 PO formalism. However, they are not accurate enough to repro445 duce the full dependence on the incidence angle. For example, 446 it is shown in Fig. 6 that the evolution with the incidence angle 447 is not merely sinusoidal as suggested by (44), although it is a 448 good approximation at the lowest angles. This loss of accuracy 449 in using the GO-like expression of the central frequency mainly 450 arises from the difference between the PO and GO models in 451 estimating the NRCS. As it is well known, the GO model has a 452 more restricted domain of validity than the PO as the incidence 453 angle is increased. Since the signal intensity enters in the 454 calculation of the central frequency (see parameter $\left\langle\left|D_{t}\right|^{2}\right\rangle$ in 455 (29) which is proportional to the NRCS), this explains the slight 456 discrepancy of the mean Doppler shifts calculated after these 457 two models at a higher incidence. Note that this is not the case 458 for the correlation time estimated in Section III, which is robust 459 to the use of the GO model since it involves only the normalized 460 (i.e., divided by the NRCS) spatiotemporal correlation function 461 of the backscattered signal. An interesting consequence of the 462 sinusoidal variation in azimuth for the central frequency is that 463 a robust joint estimator of both the wind direction $\left(\phi_{w}\right)$, and 464 the maximum central frequency $\left(F_{c}\right)$ can be built from any 465 azimuthal sampling of the instantaneous residual Doppler shift 466 at a fixed incidence angle. This could be achieved, for example, 467 using a maximum-likelihood estimator, as was done in [32] in 468 the context of NRCS azimuthal airborne data with a strong 469 level of noise. However, the construction and evaluation of this 470 estimator goes beyond the scope of this paper and is left for 471 further research.

\section{B. Influence of a Constant Surface Drift}

473 In the last decade, it has been demonstrated with spaceborne 474 [5] and airborne [30] data that the Doppler shift of the radar echo carries a clear signature of the surface current once cor- 475 rected from the wind-wave-induced Doppler anomaly. These 476 results are, however, limited to medium incidences and strong 477 currents, and we will address here the issue of low incidence 478 and small currents. The additional Doppler frequency shift 479 induced by a surface drift of norm $U$ oriented in the horizontal 480 plane with an azimuth angle $\phi_{U}$ with respect to the $x$-axis 481 (see Fig. 2) is of the form

$f_{\text {drift }}=-\frac{2}{\lambda_{0}} U \sin \theta \cos \left(\phi-\phi_{U}\right)=-F_{U} \cos \left(\phi-\phi_{U}\right)$.

The resulting total Doppler shift is given by (assuming no 483 hydrodynamical interactions between surface drift and wave 484 motion)

$$
F=-F_{c} \cos \left(\phi-\phi_{w}\right)-F_{U} \cos \left(\phi-\phi_{U}\right)=-F_{m} \cos (\phi-\Phi)
$$

where $F_{m}$ and $\Phi$ are a combination of the Doppler shift 486 parameters associated to wave motion and surface drift

$$
\left\{\begin{array}{l}
F_{m}=\sqrt{F_{c}^{2}+F_{U}^{2}+2 F_{c} F_{U} \cos \left(\phi_{w}-\phi_{U}\right)} \\
\tan \Phi=\frac{F_{c} \sin \phi_{w}+F_{U} \sin \phi_{U}}{F_{c} \cos \phi_{w}+F_{U} \cos \phi_{U}} .
\end{array}\right.
$$

The four unknown parameters $F_{C}, F_{U}, \phi_{w}$, and $\phi_{U}$ cannot be 488 recovered from the sole knowledge of $F_{m}$ and $\Phi$. Therefore, 489 the inversion of the surface current magnitude and direction re- 490 quires independent knowledge and compensation of the wave- 491 induced surface velocity, as was done in [30] with the airborne 492 ATI SAR data. Furthermore, even in the most favorable case, 493 where the surface drift is oriented in the direction of the 494 azimuthal look of the radar $\left(\phi_{U}=\phi\right)$, we find $F_{U} / F_{c} \simeq 495$ $0.4 \mathrm{~s} \cdot \mathrm{m}^{-1} U$ in Ka-band and $\simeq 0.3 \mathrm{~s} \cdot \mathrm{m}^{-1} U$ in Ku-band at 496 $10 \mathrm{~m} \cdot \mathrm{s}^{-1}$ wind speed, regardless of the incidence angle. 497 Hence, given the level of dispersion of the residual Doppler 498 shift, the relative variation induced by the surface drift seems 499 too small ( $4 \%$ for $U=10 \mathrm{~cm} \cdot \mathrm{s}^{-1}$ ) to allow for an estimation 500 of the latter in the case of moderate small-scale surface currents, 501 even if the wave-induced Doppler anomaly is known from some 502 other instrument or a geophysical model function (such as [28]). 503

\section{Potential Applications to Forthcoming Spaceborne Missions}

This study was primarily motivated by the need to estimate 506 the loss of resolution and ground cells shifts induced by wave 507 motion for the SWOT Ka interferometer (Karin, [33]). In its 508 Low Rate mode over the ocean surface, the nominal resolution 509 is expected to be $1 \mathrm{~km}^{2}$ after averaging of the $250-\mathrm{m}$ on-board 510 unfocused SAR resolution at a PRF of $4420 \mathrm{~Hz}$ with 2-ms 511 integration time. Our analysis has shown that with such a value, 512 the integration time remains larger than the correlation time, 513 which has been found on the order of $1 \mathrm{~ms}$ so that the mul- 514 tilook averaging process is efficient in processing independent 515 samples.

However, the wave-induced Doppler shifts are not negligible 517 and have to be considered in the algorithms used to estimate 518 ocean surface parameters from on-board radar altimeter signals. 519 
520 Indeed, if we consider the configuration of SWOT mission and, 521 for example, an incidence angle of $3^{\circ}$ in the upwind direction 522 for a wind speed of $10 \mathrm{~m} \cdot \mathrm{s}^{-1}$ at $10 \mathrm{~m}$, the mean Doppler shift 523 is of $40 \mathrm{~Hz}$ (see Fig. 6). This Doppler shift, if not corrected 524 for, induces a shift of the ground cells location of about $20 \mathrm{~m}$ 525 (using a simple relationship between Doppler frequency and 526 ground location), which may be not negligible for the ocean 527 height estimation.

528 In the case of crosswind direction, the mean Doppler shift 529 was found to be zero. Nevertheless, for all wind conditions, the 530 dispersion of the Doppler shift is always not null and for a wind 531 speed of $10 \mathrm{~m} \cdot \mathrm{s}^{-1}$ the ground cells location shift can reach $53250 \mathrm{~m}$ whatever the wind direction and the incidence angle.

533 It should be noted that the aforementioned conclusions have 534 considered the mean Doppler shift and its dispersion value, and 535 they should be moderated at least for the cases of null mean 536 Doppler shifts. Indeed, in that case, we can consider that the 537 dispersion value of the shift is a possible value that does not 538 systematically occur and that the shift in the ground cells loca539 tion is random. We can anticipate that the impact of this random 540 shift on the ground cells location is an additional noise on the 541 range and, hence, the ocean height estimate. We recommend 542 that further assessment of the impact of the aforementioned 543 findings on the present algorithms being designed for SWOT 544 mission be performed.

545 In the case of the nadir Doppler altimetry in Ku-band as 546 for CryoSat-2, Sentinel-3, and Jason-6, the incidence angle is 547 zero; and the mean value of the Doppler shift is always zero. 548 However, the dispersion value of the Doppler shift is, again, not 549 null as for the SWOT mission case. For the case of Ku-band, 550 the dispersion term is of about $40 \mathrm{~Hz}$ inducing a possible 551 ground cell shift of approximately $50 \mathrm{~m}$. This may be again 552 not negligible and could increase the ocean height estimation 553 noise. This should be carefully analyzed in the future.

554 Another conclusion of the present study is that the instan555 taneous residual Doppler shift distribution at low incidence 556 can be used as a proxy of wind speed through its dispersion 557 parameter rather than its centroid frequency, whereas the wind 558 direction can be, in principle, estimated from its azimuthal vari559 ations. While this is certainly not the optimal way to estimate 560 the wind vector, as compared with conventional scatterome561 ters, this information could be used in complement to another 562 sensor. For example, this supports the concept of azimuthally 563 scanning radars in the range of $0^{\circ}-15^{\circ}$ of incidence, such as 564 the SWIM instrument of the CFOSAT mission [34], which has 565 been primarily devised for wave spectra estimation but could 566 be also used in an upgraded coherent version for simultaneous 567 wind estimation. Another interesting potential application is the 568 estimation of the wind vector in extreme weather conditions. 569 We did not push the model to very high wind speed, at which 570 the spectral surface models, as well as the PO/GO scattering 571 models, become questionable. However, it is known that the 572 altimeter cross section remains sensitive to wind speed even 573 by strong sea states. If the observed trends remain true at 574 higher wind speed, the Doppler-based wind estimation at low 575 incidence could be a valuable tool for wind vector estimation in 576 high wind conditions. As to the surface current, its component 577 along the radar direction at low incidence is too weak to be esti- mated from a Doppler shift. However, even at large incidences, 578 where its effect is larger, the contribution of surface current to 579 the Doppler shift is drowned in the Doppler anomaly induced 580 by wave motion, which is the dominant contribution. Hence, 581 the elimination of this Doppler anomaly is a first necessary step 582 in view of any estimation of the surface current. For this, it 583 might be interesting to combine multiple sensors at low and 584 high incidences to better characterize the long waves and their 585 Doppler anomaly.

586

\section{CONCLUSION}

587

We have investigated the decorrelation time and the instan- 588 taneous residual Doppler shift distribution of the sea surface 589 backscattered signal in the framework of the PO and GO at 590 low incidence. A simple expression has been found for the 591 decorrelation time as a function of the main sea state parameters 592 (peak wavenumber and significant wave height), in excellent 593 agreement with the rare data available in the literature. For the 594 SWOT mission, the decorrelation time is consistent with the 595 multilook averaging process. In the framework of the PO, we 596 have proposed a TDE for the instantaneous residual Doppler 597 shift distribution due to wave motion resulting in a simple 598 analytic expression in terms of a nonstandardized Student's 599 $t$-distribution and the statistical parameters of the surface. The 600 limitation of the TDE, namely, the inability to separate positive 601 and negative Doppler shifts, is overcome to produce a full two- 602 sided Doppler spectrum. The evolution of the mean and dis- 603 persion of the Doppler shift has been investigated with respect 604 to the sea states and the scattering angles. The mean Doppler 605 shift has a weak sensitivity to wind speed but its dispersion 606 dramatically increases with the latter, suggesting that the width 607 of the Doppler shift distribution is a better proxy than its mean 608 value for wind speed estimation at low incidence. The situation 609 is opposite when it comes to the influence of the scattering 610 geometry as the mean Doppler frequency mainly depends on 611 the scattering angles, whereas the dispersion parameter mainly 612 depends on the surface roughness. We have further shown that 613 the mean Doppler frequency follows a sinusoidal variation in 614 azimuth with respect to the wind direction, which could be 615 used to devise a robust estimator of its direction. The influence 616 of additional surface currents has been evaluated. Their impact 617 on the mean Doppler shift cannot be separated from the wave- 618 induced Doppler anomaly unless the latter is known and accu- 619 rately compensated by some other means. However, even in this 620 case, a systematic inversion seems out of reach as the relative 621 contribution of the surface current to the mean Doppler shift 622 is small, whereas the dispersion of the wave-induced Doppler 623 shift is large. Finally, it has been recommended that the derived 624 distribution of Doppler shift be further considered in the SWOT 625 mission to further assess the impact on the estimated parameters 626 and possibly improve the algorithms accordingly.

627

\section{APPENDIX A}

628

In the limit of short radar-wavelength the coherent term 629 $e^{-Q_{z}^{2} \rho_{0}}$ in integral (8) vanishes and the spatiotemporal corre- 630 lation function of the surface $(\rho)$ can be approximated by its 631 


$$
\begin{aligned}
& C_{\mathrm{GO}}(t)=e^{\overbrace{}^{-\frac{Q_{z}^{2}}{2}\left[s_{t t}^{2}-\frac{s_{y y}^{2} s_{x t}^{2}-2 s_{x y}^{2} s_{x t} s_{y t}+s_{x x}^{2} s_{y t}^{2}}{\Sigma^{2}}\right]} t^{2} \overbrace{e^{-i\left[Q_{H x} x_{0}(t)+Q_{H y} y_{0}(t)\right]}}^{T_{\mathrm{GO}}(t)(13)} \mathbb{M}_{\mathrm{GO}}(t)(16)} \\
& \times \underbrace{\frac{1}{\pi} \frac{|\mathbb{K}|^{2}}{Q_{z}^{2}} \int_{-\infty}^{\infty} \int_{-\infty}^{\infty} e^{-i\left[Q_{H x} x+Q_{H y} y\right]} e^{-\frac{Q_{z}^{2}}{2}\left[s_{x x}^{2} x^{2}+2 s_{x y}^{2} x y+s_{y y}^{2} y^{2}\right]} d x d y}_{\sigma_{\mathrm{GO}}^{0}}
\end{aligned}
$$

632 second-order Taylor expansion about the origin (the depen633 dence to the wind direction $\phi_{w}$ is implicit in the following 634 expressions):

$$
\rho(0,0,0)-\rho(x, y, t) \simeq s_{x x}^{2} \frac{x^{2}}{2}-s_{x t} x t+s_{x y}^{2} x y-s_{y t} y t+s_{y y}^{2} \frac{y^{2}}{2}
$$

635 where the coefficients are defined by (11) and (15). Denoting $636 Q_{H x}$ and $Q_{H y}$ the projections of the horizontal component of 637 the Ewald vector $\left(\mathbf{Q}_{\mathbf{H}}\right)$ on the $x$ - and $y$-axis, respectively, this 638 leads to the following approximation of the correlation integral:

$$
\begin{gathered}
C_{\mathrm{GO}}(t)=\frac{1}{\pi} \frac{|\mathcal{K}|^{2}}{Q_{z}^{2}} e^{-\frac{Q_{z}^{2}}{2} s_{t t}^{2} t^{2}} \int_{-\infty}^{\infty} \int_{-\infty}^{\infty} e^{-i\left[Q_{H x} x+Q_{H y} y\right]} \times \cdots \\
\cdots e^{-\frac{Q_{z}^{2}}{2}\left[s_{x x}^{2} x^{2}-2 s_{x t} x t+2 s_{x y}^{2} x y-2 s_{y t} y t+s_{y y}^{2} y^{2}\right]} d x d y
\end{gathered}
$$

639 Using the standard formula for the Fourier transform of a 640 noncentered 2-D Gaussian function, we obtain, (51) shown at 641 the top of the page. leading to expression (9).

\section{ACKNOWLEDGMENT}

643 The authors would like to thank M. Saillard for useful 644 comments.

\section{REFERENCES}

1] D. R. Thompson, "Calculation of microwave Doppler spectra from the ocean surface with a time-dependent composite model," in Radar Scattering from Modulated Wind Waves. New York, NY, USA: SpringerVerlag, 1989, pp. 27-40.

[2] W. J. Plant, "A model for microwave Doppler sea return at high incidence angles: Bragg scattering from bound, tilted waves," J. Geophys. Res., Oceans, vol. 102, no. C9, pp. 21 131-21 146, 1997.

[3] V. U. Zavorotny and A. G. Voronovich, "Two-scale model and ocean radar Doppler spectra at moderate-and low-grazing angles," IEEE Trans. Antennas Propag., vol. 46, no. 1, pp. 84-92, Jan. 1998.

[4] R. Romeiser and D. Thompson, "Numerical study on the along-track interferometric radar imaging mechanism of oceanic surface currents," IEEE Trans. Geosci. Remote Sens., vol. 38, no. 1, pp. 446-458, Jan. 2000.

[5] B. Chapron, F. Collard, and F. Ardhuin, "Direct measurements of ocean surface velocity from space: Interpretation and validation," J. Geophys. Res., Oceans, vol. 110, no. C7, pp. 76-92, Jul. 2005.

[6] A. A. Mouche, B. Chapron, N. Reul, and F. Collard, "Predicted Doppler shifts induced by ocean surface wave displacements using asymptotic electromagnetic wave scattering theories," Waves Random Complex Media, vol. 18, pp. 185-196, 2008.

[7] F. Nouguier, C.-A. Guérin, and G. Soriano, "Analytical techniques for the Doppler signature of sea surfaces in the microwave regime-I: Linear surfaces," IEEE Trans. Geosci. Remote Sens., vol. 49, no. 12, pp. 4856-4864, Dec. 2011

670 [8] F. Nouguier, C.-A. Guérin, and G. Soriano, "Analytical techniques for

671 the Doppler signature of sea surfaces in the microwave regime-II: Non-

672 linear surfaces," IEEE Trans. Geosci. Remote Sens., vol. 49, no. 12,

673
[9] X. Li and X. Xu, "Scattering and Doppler spectral analysis for two- 674 dimensional linear and nonlinear sea surfaces," IEEE Trans. Geosci. 675 Remote Sens., vol. 49, no. 2, pp. 603-611, Feb. 2011.

[10] Y. Wang, Y. Zhang, M. He, and C. Zhao, "Doppler spectra of microwave 677 scattering fields from nonlinear oceanic surface at moderate-and low- 678 grazing angles," IEEE Trans. Geosci. Remote Sens., vol. 50, no. 4, 679 pp. 1104-1116, Apr. 2012.

[11] F. Fois, P. Hoogeboom, F. Le Chevalier, and A. Stoffelen, "An analytical 681 model for the description of the full-polarimetric sea surface Doppler 682 signature," J. Geophys. Res., Oceans, vol. 120, no. 2, pp. 988-1015, 2015. 683 doi: http://dx.doi.org/10.1002/2014JC010589.

[12] J. V. Toporkov and G. S. Brown, "Numerical simulations of scattering 685 from time-varying, randomly rough surfaces," IEEE Trans. Geosci. 686 Remote Sens., vol. 38, no. 4, pp. 1616-1624, Jul. 2000.

[13] J. Johnson and R. Burkholder, "Coupled canonical grid/discrete dipole ap- 688 proach for computing scattering from objects above or below a rough in- 689 terface," IEEE Trans. Geosci. Remote Sens., vol. 39, no. 6, pp. 1214-1220, 690 Jun. 2001

[14] A. R. Hayslip, J. T. Johnson, and G. R. Baker, "Further numerical stud- 692 ies of backscattering from time evolving non-linear sea surfaces," IEEE 693 Trans. Geosci. Remote Sens., vol. 41, no. 10, pp. 2287-2293, Oct. 2003. 694

[15] G. Soriano, M. Joelson, and M. Saillard, "Doppler spectra from a two- 695 dimensional ocean surface at l-band," IEEE Trans. Geosci. Remote Sens., 696 vol. 44, no. 9, pp. 2430-2437, Sep. 2006

[16] J. V. Toporkov and M. A. Sletten, "Statistical properties of low- 698 grazing range-resolved sea surface backscatter generated through two- 699 dimensional direct numerical simulations," IEEE Trans. Geosci. Remote 700 Sens., vol. 45, no. 5, pp. 1181-1197, May 2007.

[17] C.-S. Chae and J. T. Johnson, "A study of sea surface range-resolved 702 Doppler spectra using numerically simulated low-grazing-angle backscat- 703 ter data," IEEE Trans. Geosci. Remote Sens., vol. 47, no. 6, pp. 3452- 704 3460, Jun. 2013.

[18] D. Miret, G. Soriano, F. Nouguier, P. Forget, M. Saillard, and C.-A. Guérin, 706 "Sea surface microwave scattering at extreme grazing angle: numerical 707 investigation of the Doppler shift," IEEE Trans. Geosci. Remote Sens., 708 vol. 52, no. 11, pp. 7120-7129, Nov. 2014.

[19] M. H. Sharqawy, J. H. Lienhard V, and S. M. Zubair, "Thermophysi- 710 cal properties of seawater: A review of existing correlations and data," 711 Desalination Water Treat., vol. 16, pp. 354-380, Apr. 2010.

[20] T. Elfouahily, B. Chapron, K. Katsaros, and D. Vandemark, "A unified 713 directional spectrum for long and short wind-driven waves," J. Geophys. 714 Res., vol. 102, no. C7, pp. 15 781-15 796, Jul. 1997.

[21] M. S. Longuet-Higgins, D. E. Cartwright, and N. D. Smith, "Observations 716 of the directional spectrum of sea waves using the motions of a floating 717 buoy," in Ocean Wave Spectra. Englewood Cliffs, NJ, USA: Prentice- 718 Hall, 1963, pp. 111-136.

[22] W. J. Plant, "A stochastic, multiscale model of microwave backscatter 720 from the ocean," J. Geophys. Res., Oceans, vol. 107, no. C9, pp. 3-1-3-21, 721 Sep. 2002. [Online]. Available: http://dx.doi.org/10.1029/2001JC000909 722

[23] T. Elfouhaily and C.-A. Guérin, "A critical survey of approximate scatter- 723 ing wave theories from random rough surfaces," Waves Random Complex 724 Media, vol. 14, no. 4, pp. 1-40, 2004.

[24] P. Beckmann and A. Spizzichino, The Scattering of Electromagnetic 726 Waves From Rough Surfaces. Norwood, MA, USA: Artech House, 1987. 727

[25] R. D. Chapman, B. L. Gotwols, and R. E. Sterner, "On the statistics of 728 the phase of microwave backscatter from the ocean surface," J. Geophys. 729 Res., Oceans, vol. 99, no. C8, pp. 16 293-16301, 1994. doi: http://dx.doi. 730 org/10.1029/94JC01111.

[26] S. Madsen, "Estimating the Doppler centroid of SAR data," IEEE Trans. 732 Aerosp. Electron. Syst., vol. 25, no. 2, pp. 134-140, Mar. 1989.

[27] R. Baxley, B. Walkenhorst, and G. Acosta-Marum, "Complex Gaussian 734 ratio distribution with applications for error rate calculation in fading 735 channels with imperfect CSI," in Proc. IEEE GLOBECOM, Dec. 2010, 736 pp. 1-5. 
738 [28] A. A. Mouche et al., "On the use of Doppler shift for sea surface wind retrieval from SAR," IEEE Trans. Geosci. Remote Sens., vol. 50, no. 7 , pp. 2901-2909, Jul. 2012.

29] K. F. Dagestad et al., "Wind retrieval from synthetic aperture Radar-An overview," Eur. Space Agency, ESA, Paris, France, 2013.

30] A. C. H. Martin, C. Gommenginger, J. Marquez, S. Doody, V. Navarro, and C. Buck, "Wind-wave-induced velocity in ATI SAR ocean surface currents: First experimental evidence from an airborne campaign," J. Geophys. Res., Oceans, vol. 121, no. 3, p. 1640-1653, 2016.

31] P. Forget, M. Saillard, C.-A. Guérin, J. Testud, and E. L. Bouar, "On the use of x-band weather radar for wind field retrieval in coastal zone," J. Atmosp. Ocean. Technol., vol. 33, no. 5, pp. 899-917, May 2016.

32] Z. Guerraou, S. Angelliaume, L. Rosenberg, and C.-A. Guérin, "Investigation of azimuthal variations from $\mathrm{x}$-band medium grazing angle sea clutter," IEEE Trans. Geosci. Remote Sens., vol. 54, no. 10, pp. 6110-6118, Oct. 2016.

33] M. Durand, L. L. Fu, D. P. Lettenmaier, D. E. Alsdorf, E. Rodriguez, and D. Esteban-Fernandez, "The surface water and ocean topography mission: Observing terrestrial surface water and oceanic submesoscale eddies," Proc. IEEE, vol. 98, no. 5, pp. 766-779, May 2010.

34] C. Tison et al., "Directional wave spectrum estimation by swim instrument on CFOSAT," in Proc. IEEE Int. Geosci. Remote Sens. Symp., Jul. 2009, vol. 5, pp. V-312-V-315.

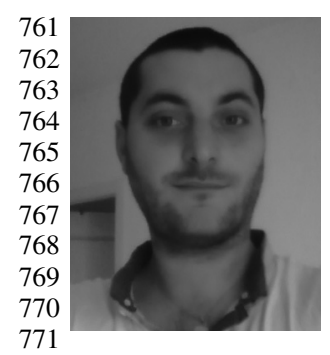

Olivier Boisot was born in Toulon, France, in 1987. He received the M.S. degree in physics and engineering sciences and the Ph.D. degree in physics from the University of Toulon, La Garde, France, in 2012, respectively 2015 .

He was with the Mediterranean Institute of Oceanography, University of Toulon, La Garde, France, the Centre National d'Études Spatiales (CNES), Toulouse, France, and Collecte Localisation Satellite (CLS), Toulouse, France, on electromagnetic scattering modelization from rough surfaces and its appli772 cations to the Surface Water and Ocean Topography mission. He is currently 773 a Postdoctoral Fellow with the Office National d'Études et de Recherches 774 Aéspatiales (ONERA), Salon-de-Provence, France, and is working on the 775 detection and characterization of chemical products and oil on the ocean surface 776 from synthetic aperture radar measurements in microwave bands.
Laïba Amarouche, photograph and biography not available at the time of 777 publication.

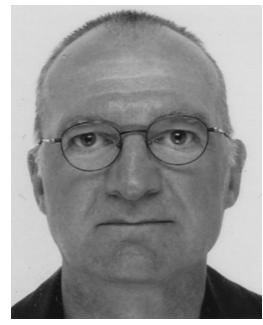

\section{Jean-Claude Lalaurie}

Since 1991, he has been an Engineer with the 780 AQ8 Centre National d'Études Spatiales (CNES) and 781 started his career in microwave instrument design 782 and development [MMIC components for radio 783 AQ9 communication equipment and synthetic aperture 784 radar (SAR) systems]. In 1999, he started special- 785 izing in electric and magnetic field instruments for 786 plasma characterization. He was involved in the de- 787 velopment of several instruments, including Deme- 788 ter, Venus Express, and SWARM. Since 2010, he 789 has been on water surface backscattering characterization, interferometric SAR 790 height bias estimation, and acquisition of near-field and airborne Ka-band data 791 in the framework of the Surface Water and Ocean Topography project.

792
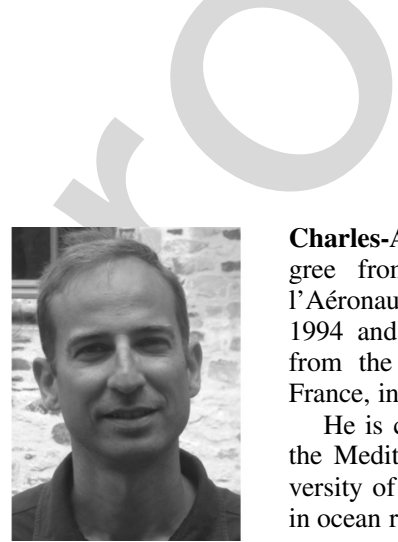

Charles-Antoine Guérin received the B.Eng. de- 793 gree from the Ecole Nationale Supérieure de 794 l'Aéronautique et de l'Espace, Toulouse, France, in 795 1994 and the Ph.D. degree in theoretical physics 796 from the University of Aix-Marseille, Marseille, 797 France, in 1998.

798

$\mathrm{He}$ is currently a Professor and Researcher with 799 the Mediterranean Institute of Oceanography, Uni- 800 versity of Toulon, La Garde, France. He specializes 801 in ocean remote sensing.

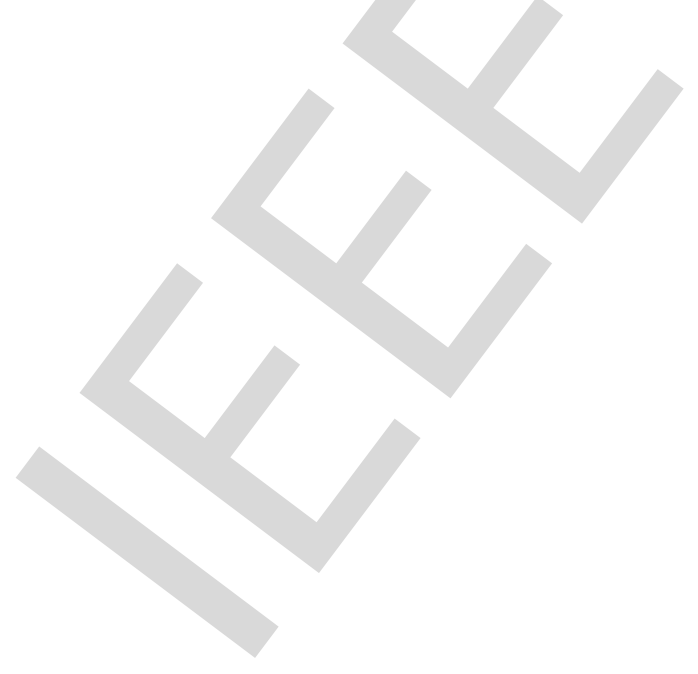

